\title{
Novel Modification of HeartMate 3 Implantation
}

\author{
Hadley K. Wilson, MD, Benjamin E. Haithcock, MD, and Thomas G. Caranasos, MD \\ University of North Carolina School of Medicine, Chapel Hill, North Carolina; and Division of Cardiothoracic Surgery, Department of \\ Surgery, University of North Carolina Hospitals, Chapel Hill, North Carolina
}

We have modified the HeartMate 3 (Abbott, Abbott Park, IL) implantation technique to better suit our patient population. This modification optimizes the placement of the HeartMate 3 sewing cuff and allows passage of the suture transmurally from endocardium to epicardium in a "cut

$\mathrm{T}^{\mathrm{s}}$ he HeartMate 3 left ventricular assist device (LVAD) system (Abbott, Abbott Park, IL) has become an effective and popular LVAD, both nationally and at our institution. The standard implantation technique recommended by manufacturer uses horizontal mattress sutures passed from a continuous felt strip or through a large felt pledget into the ventricular myocardium and then through the sewing ring in a "sew then cut" method. ${ }^{1,2}$ After the ring is secured, the coring device is used to remove a core of ventricular myocardium to allow for placement of the inflow cannula.

Other modifications exist for apical implantation. This report details a "cut then sew" method using endocardial-to-epicardial suture placement. This method was developed at our institution with excellent outcomes. While this technique is not meant to replace other methods of implantation, we have found it particularly useful in patients at our institution with thin, friable ventricular myocardium.

\section{Technique}

The heart is exposed through a standard median sternotomy. We first tunnel the driveline before any heparin is administered. The patient is systemically heparinized and is cannulated for bypass using ascending aortic and standard right atrial cannulas. Cardiopulmonary bypass is then initiated, and the left ventricular (LV) apex is elevated into the field using laparotomy sponges packed in the pericardium. The LV apex and inflow cannula position are determined by digital palpation with transesophageal echocardiography guidance.

We then make an incision with an 11 blade scalpel. A Coude Foley catheter is placed through this incision, and the coring device is advanced over the catheter in the previously determined direction and position. The

Accepted for publication Jun 18, 2020.

Address correspondence to Dr Wilson, Department of Surgery, University of North Carolina Hospitals, 101 Manning Dr, Chapel Hill, NC 27514; email: hadley.wilson@unchealth.unc.edu. then sew" technique. We believe this affords a superior seal and protection from tearing friable myocardium.

LV apical core is removed and sent to pathology. Trabeculations that may obstruct the inflow cannula are excised.

Using a 2-0 Ethibond suture (Ethicon, Somerville, NJ) on an SH-2 needle loaded with 0.375 -inch $\times 0.1875$ inch $\times 0.0625$-inch felt pledgets, we place 12 (or as many as necessary) interrupted horizontal mattress sutures passing from endocardium to epicardium. We do not use large hand-cut pledgets to prevent overcrowding within the LV cavity. Once all of the sutures are in place, these are then passed through the sewing ring and tied down.

The HeartMate 3 is then brought onto the field and connected to the sewing ring. We place the LV vent to the outflow of the HeartMate 3 to remove air before we connect the outflow graft. The outflow graft anastomosis is constructed in the standard fashion. This technique can also be used if using an "outflow first" technique, performing the outflow graft anastomosis before initiating cardiopulmonary bypass.

\section{Comment}

We have found this technique with endocardial-toepicardial sutures to be safe and effective. Because of improved visualization and the more direct suture path from endocardium to epicardium, we have found this method to be particularly useful in delicate, friable ventricular apices. In our experience, the straight path of the suture through the ventricular myocardium and distribution of force using intraventricular pledgets optimizes the HeartMate 3 sewing cuff to prevent tearing of friable left ventricular apices. The felt sewing ring for the HeartMate 3 acts as a large pledget for the epicardial side and, in combination with intraventricular pledgets, distributes the tension of the sutures more evenly to prevent ventricular injury.

Criticism of this technique could be the potential for pledget loss, device malfunction, or thrombus formation. We have implanted more than 100 HeartMate 3 LVADs at our institution using this technique, of which 10 of those 


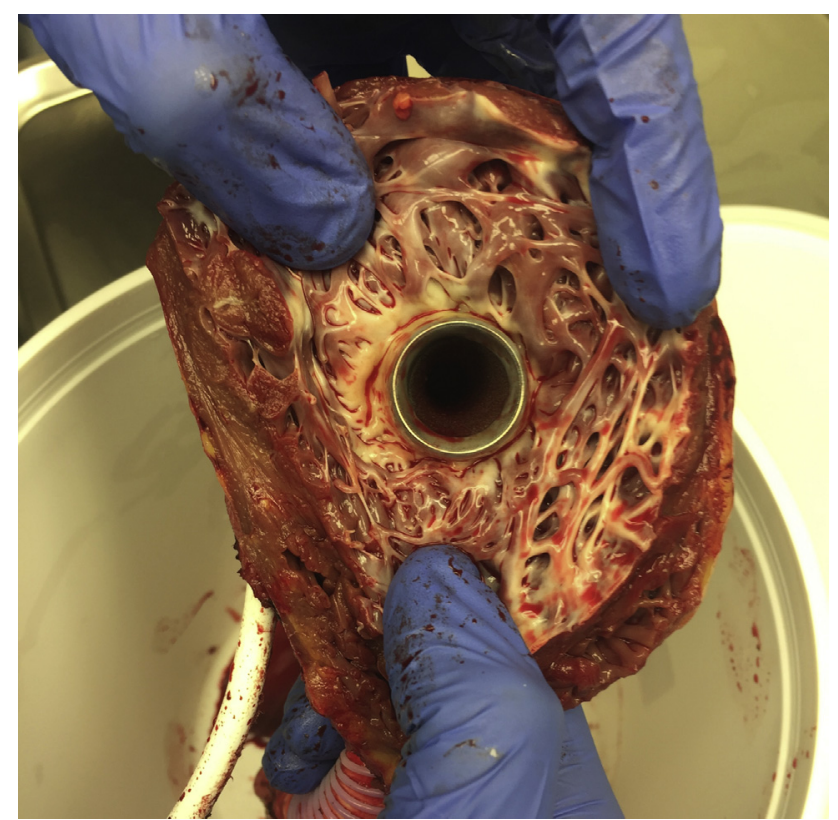

Figure 1. Pledgets well incorporated into the endocardium of the explanted heart at the time of transplant.

patients have gone on to transplant. At the time of recipient cardiectomy, we have evaluated the native heart and HeartMate 3 and found excellent endothelialization. The pledgets are well incorporated into the endocardium (Figures 1, 2).

In our experience, we have had no pledget-related complications and have successfully navigated a myriad of ventricular apical anatomy. We do not believe that our technique is superior to others, but instead, we offer an alternative method to be used at the discretion of the implanting surgeon as circumstances dictate. While we believe this method to be safe in our experience, further study will help delineate the risk of pledget loss, pump thrombosis, and panus ingrowth.

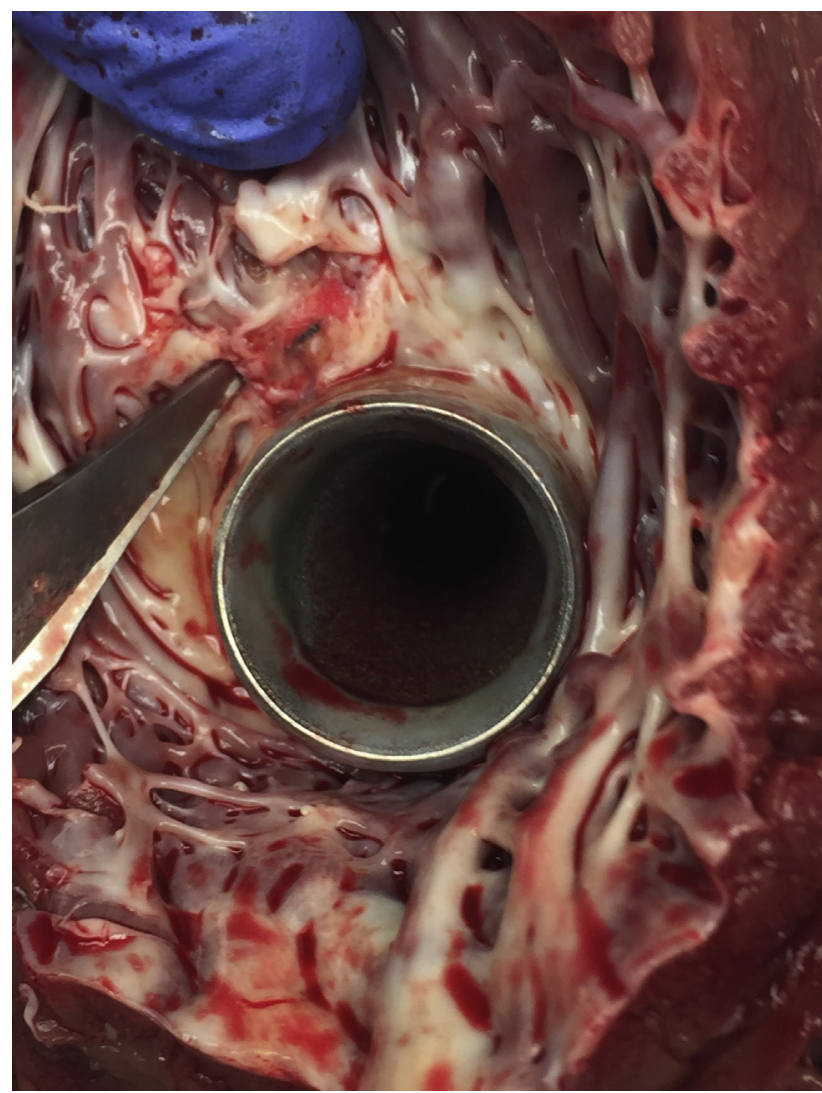

Figure 2. Endocardium removed to expose well-incorporated pledgets.

\section{References}

1. Netuka I. HeartMate 3 left ventricular assist system implantation technique: the devil is in the detail. Interact Cardiovasc Thorac Surg. 2018;27:946-949.

2. Beyersdorf F, Scheumann J, Siepe M. Implantation of the HeartMate 3-description of the surgical technique. Oper Tech Thorac Cardiovasc Surg. 2017;22:173-185. 